\title{
Role of Peroral Cholangioscopy for Diagnosis and Staging of Biliary Tumors
}

\author{
Pedro Pereira ${ }^{a, b}$ Sancha Santos $^{b}$ Rui Morais ${ }^{a}$, Rui Gaspar ${ }^{a, b}$ \\ Eduardo Rodrigues-Pinto ${ }^{a, b}$ Filipe Vilas-Boas ${ }^{a, b}$ Guilherme Macedo ${ }^{a, b}$ \\ ${ }^{a}$ Department of Gastroenterology, Centro Hospitalar Universitário São João, Porto, Portugal; b Faculty of Medicine, \\ University of Porto, Porto, Portugal
}

\section{Keywords}

Endoscopic retrograde cholangiopancreatography · Peroral cholangioscopy · Biliary strictures · Cholangiocarcinoma

\begin{abstract}
Objectives: Peroral cholangioscopy (POC) has shown to be a useful diagnostic procedure in the evaluation of biliary strictures; however, data regarding its role on preoperative staging are scarce. The aim of this study was to evaluate POC role in the diagnosis and preoperative intraductal staging of perihilar cholangiocarcinoma (CCA). Methods: Retrospective study that included all patients who underwent POC with SpyGlass ${ }^{\mathrm{TM}}$ Direct Visualization System for the diagnosis of biliary strictures or for preoperative evaluation of extrahepatic biliary tumors, between 2015 and 2019, in a single tertiary center. Results: Forty-three patients were included, 63\% male with a median age of 62 years. Thirty-eight (88.3\%) underwent POC due to indeterminate biliary strictures, 3 (7\%) due to bile duct filling defect, and 2 (4.7\%) for intraductal staging of perihilar CCA. In the follow-up, a final diagnosis of malignancy was established in $56 \%$ of the patients. Visual impression accuracy with SpyGlass was 95.1\% (with 100\% sensitivity and $89.5 \%$ specificity). SpyBite biopsies accuracy was $80.5 \%$ (63.6\% sensitivity and $100 \%$ specificity). In the 19 patients with a final perihilar CCA diagnosis, intraductal evaluation with SpyGlass altered anatomic classification (Bismuth-Corlette) defined by previous imagiologic findings in
\end{abstract}

\section{KARGER}

๑) 2020 S. Karger AG, Basel
$8(42.1 \%)$ patients. Alteration in anatomic classification changed therapeutic approach in 4 (21\%). Conclusions: POC use for evaluating intraductal spread in potentially resectable perihilar CCA can detect more extensive and change surgical management. In the future, preoperative staging of perihilar CCA with POC combined with imagiologic evaluation of vascular extension of the lesions may optimize surgical results.

(c) 2020 S. Karger AG, Basel

\section{Introduction}

Biliary strictures, which are a frequent finding in clinical practice, can be caused by a set of benign and malignant conditions, each requiring a specific treatment approach $[1,2]$. Malignant bile duct strictures are usually due to pancreatic adenocarcinoma or cholangiocarcinoma (CCA). The latter should always be considered in mid and proximal extrahepatic bile duct strictures, with the main focus being to rule out this entity [3].

Endoscopic retrograde cholangiopancreatography (ERCP) is an important tool in patients presenting with a biliary stricture. However, ERCP tissue sampling techniques, such as brush cytology, "blind" intraductal forceps biopsy, or a combination of both, are hindered by low sensitivity [4]. Thus, up to $20 \%$ of biliary strictures remain indeterminate after preoperative evaluation, including a basic 
laboratory work up, abdominal imaging, and ERCP with biliary sampling, and require surgical resection with pathologic evaluation for a definitive diagnosis [5]. Moreover, up to one-quarter of suspected malignant strictures are determined to be benign at the time of surgical resection [5].

Peroral cholangioscopy (POC), allowing direct visualization of the biliary tract with targeted biopsy of suspicious lesions, is a promissory diagnostic tool in those cases in which a definite diagnosis cannot be obtained $[6,7]$. Since 1970, several systems have been created; however, widespread adoption of POC has been hindered by technological obstacles [8]. Single operator POC using the SpyGlass ${ }^{\mathrm{TM}}$ Direct Visualization System (SGDVS; Boston Scientific Corp., Natick, MA, USA) was introduced in 2007. The first-generation system (SpyGlass ${ }^{\mathrm{TM}}$ ) consists of capital equipment, including a light source, monitor, access and delivery catheter (SpyScope), and an optical probe (SpyGlass) with an irrigation pump. This optical system was later replaced for a video system (SpyGlass ${ }^{\mathrm{TM}}$ DS) with improved image quality.

Even though the majority of CCA patients are diagnosed at late-stage disease when resection is no longer an option, surgery remains the only curative therapy. Perihilar CCA is the most frequent subtype, and the BismuthCorlette classification (BCC) was developed to guide surgical therapy [9]. In these settings, $\mathrm{BBC}$ provides an anatomic description of the tumor location and longitudinal extension in the biliary tree. Thus, diagnosing the precise mucosal tumor extension preoperatively is essential in order to limit costs and morbidity to the patient.

SGDVS can also have a role in these presurgical "mapping" of biliary lesions anticipating surgical resection. In this context, direct observation of the biliary tree can be used to identify the extent of disease and adjust clinical decision making.

Further data on the SpyGlass system are necessary to establish its role in the evaluation of patients with biliary strictures. The aim of our study was to evaluate the clinical utility of SpyGlass visual findings and SpyBite biopsy findings not only in the diagnosis of biliary strictures but also in detecting mucosal cancerous extension preoperatively in bile duct cancers.

\section{Materials and Methods}

\section{Study Design}

Retrospective study performed in a tertiary center. The study was approved by the Ethics Review Committee. As approved by the Ethics Board, informed consent was not required for this study due to its retrospective design.

\section{Patients}

We evaluated medical records from all patients, between January 2015 and February 2019, who underwent SGDVS (Boston Scientific).

All patients were initially evaluated in a multidisciplinary consultation and included in the study when there was an indeterminate biliary stricture or filling defect or uncertainty about resectability in a patient with previous diagnosis of perihilar CCA. In all patients, the purpose of POC was to establish a diagnosis and evaluate the presence of longitudinal mucosal cancer extension that could alter surgical treatment in malignant lesions. Patients with a previous diagnosis of perihilar CCA were excluded from the evaluation of POC role in the diagnosis of biliary strictures.

As a part of our institutional protocol, computed tomography and/or endoscopic ultrasound was performed before the procedure in patients with distal lesions and magnetic resonance cholangiopancreatography in perihilar lesions. In patients from other centers, imaging studies were reviewed by a trained radiologist in our multidisciplinary consultation previous to the POC examination. The time-lapse between imaging studies and POC examination was no longer than 3 weeks.

Exclusion criteria were patients medically unfit to undergo ERCP and patients submitted to POC for the treatment of difficult biliary stones.

\section{Technical Aspects of SpyGlass Cholangioscopy}

Examinations were performed by an experienced therapeutic endoscopist (annual ERCP volume load >150; with $>2,000$ lifetime ERCP performed), who visualized and performed 10 SpyGlass procedures before starting the study to obtain and build up the necessary skills and technical experience with the platform.

All procedures were performed under general anesthesia. Before the procedure, all patients received antibiotic prophylaxis (ciprofloxacin) and rectal indomethacin to prevent postprocedure pancreatitis. Biliary sphincterotomy, if not yet performed, was carried out just before the SpyGlass examination. Each procedure was performed with an Olympus TJF-160V or TJF-Q180V duodenoscope (Olympus Medical Systems, Tokyo, Japan) along with the SpyGlass and accessories (Boston Scientific Corp., Natick, MA, USA). After initial fluoroscopic biliary stenosis characterization, a guidewire was introduced into the target duct, and SpyScope was advanced proximal to the stricture. On withdrawal, careful examination of the remainder biliary tree was performed according to segmental classification (right posterior duct, right anterior duct, right hepatic duct, left hepatic duct, and common hepatic duct), and every ductal lesion was visually characterized. When a lesion was detected, confrontation with fluoroscopic imaging was performed for exact location. The intraductal involvement was determined by visual inspection. Thereafter, and if possible, biopsy specimens (at least 4) were obtained using the SpyBite forceps under SpyGlass guidance. An experienced anatomopathologist evaluated all samples for adequacy. In all cases, the stenosis accommodated the SpyScope, so there was no need to perform balloon dilatation prior to the cholangioscopy. At the end of the procedure, a stent was inserted to ensure drainage.

\section{End Points and Cholangioscopy Findings}

The primary end point was to assess the accuracy, sensitivity, and specificity of SpyGlass visual findings and SpyGlass-guided biopsy findings in the diagnosis of patients with biliary strictures, 
as well as postprocedural adverse events (AEs). Severity grade of AEs were classified according to American Society of Gastrointestinal Endoscopy lexicon for endoscopic AEs into mild, moderate, severe, and fatal [10].

Visual impression of biliary lesions were classified according to Xie et al. [11] classification as malignant if one of the following visual findings were detected: (1) dilated tortuous vessels (tumor vessels), (2) infiltrative stricture, (3) villous mass, (4) finger projections, (5) papillary projections, (6) concentric stenosis, (7) granular mucosa, and (8) irregular mucosal nodularity. Benign lesions were characterized by smooth surface mucosa without definite neovascularization and homogeneous granular mucosa without a primary mass.

All of the specimens acquired by SpyBite biopsy forceps were categorized as malignant, benign, or inconclusive for malignancy. In the statistical analysis, specimens interpreted as "suspected for malignancy" were considered positive, and specimens interpreted as "atypical" or "insufficient" were considered to be inconclusive for malignancy.

The final diagnosis of malignancy was made based on positive histopathology of SpyGlass-guided biopsy, other tissue sampling procedures such as surgical specimens, diagnostic laparoscopy with biopsy samples or progression of disease consistent with malignancy during at least a 6-month follow-up.

Patients with a final diagnosis of malignant disease underwent either curative surgery or palliative biliary stenting with or without palliative chemotherapy.

The secondary end point was to evaluate the ability of SpyGlass to detect mucosal cancerous extension preoperatively in potentially resectable perihilar CCA and the changes on surgical approach based on findings. In all final malignant cases, we compared previous imagiologic anatomic classification with that assessed with POC. Anatomic classification was based on BCC system that provides an anatomic description of the tumor location and longitudinal extension in the biliary tree. Besides, in order to conclude on possible surgical approach differences, vascular extension of the tumor was also assessed based on TNM classification [12].

\section{Statistical Analysis}

Categorical variables were described through absolute and relative frequencies and continuous variables were described as mean and SD, median, percentiles, minimum, and maximum. Hypotheses were tested about the distribution of continuous variables with nonnormal distribution, by using the nonparametric MannWhitney and Kruskal-Wallis test, depending on the nature of the hypothesis. The diagnostic accuracy, sensitivity, and specificity of SpyGlass visual impression and SpyGlass-guided biopsies were calculated and compared with those in the final diagnosis.

All data were arranged, processed, and analyzed with SPSS ${ }^{\circledR}$ version 24.0 data (Statistical Package for Social Sciences).

\section{Results}

\section{Baseline Characteristics}

During the study period, 1,092 ERCP procedures were performed for different biliary indications. Of these, 51

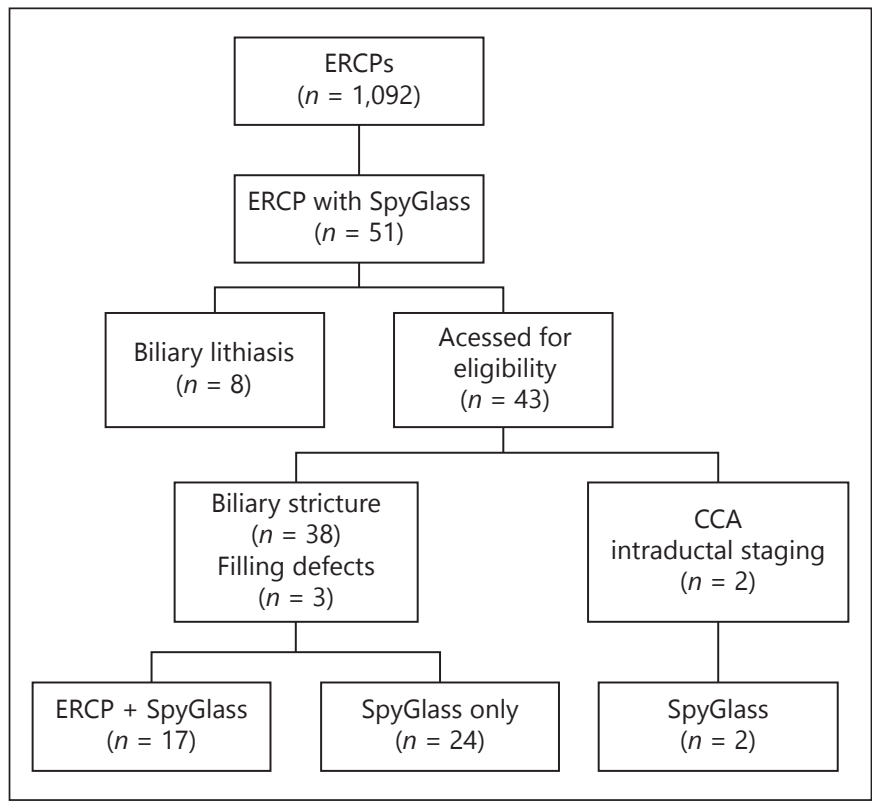

Fig. 1. Flowchart of the study. ERCP, endoscopic retrograde cholangiography; CCA, cholangiocarcinoma.

patients underwent POC with SpyGlass system. Excluding cholangioscopy for complex lithiasis, a total of 43 patients were eligible for our study (Fig. 1). The study population consisted of 27 men (62.8\%) and 16 women (37.2\%) with a mean age of $61 \pm 13$ years.

Indications for SpyGlass cholangioscopy according to imagiologic or ERCP findings were biliary strictures $(n=$ $38 ; 88.3 \%)$, filling defects $(n=3 ; 7 \%)$, and CCA intraductal staging $(n=2 ; 4.7 \%)$. Two patients $(4.7 \%)$ had previous ERCP with CCA diagnosis who needed further preoperative evaluation. Fifteen patients (34.9\%) had previous ERCP with nondiagnostic routine brush cytology, while the other 26 (60.4\%) underwent upfront SpyGlass. Mean follow-up after the procedure was $265 \pm 311$ days.

Of 43 patients who underwent POC, postprocedural AEs were registered in $8(18.6 \%)$, with $5(11.6 \%)$ pancreatitis and 3 (7\%) cholangitis. All AEs observed were considered mild and resolved with conservative measures. Table 1 summarizes patient and SGDVS characteristics.

\section{SpyGlass Cholangioscopy Findings}

Technical success, defined by the ability to progress with the SpyScope to the target lesion, was achieved in all the cases. Procedure with first-generation SpyGlass ${ }^{\mathrm{TM}}$ was performed in 7 patients, while 36 underwent SpyGlass ${ }^{\mathrm{TM}}$ DS. 
Table 1. Patient and procedure data

\begin{tabular}{ll}
\hline Total number of patients & $n=43$ \\
\hline $\begin{array}{l}\text { Age, years, mean } \pm \text { SD } \\
\quad \text { (minimum-maximum) }\end{array}$ & \\
Gender, $n(\%)$ & $61 \pm 13(22-86)$ \\
$\quad$ Male & $27(62.8)$ \\
$\quad$ Female & $16(37.2)$ \\
Indication for procedure, $n(\%)$ & \\
$\quad$ Biliary stricture & $38(88.3)$ \\
$\quad$ Filling defects & $3(7.0)$ \\
$\quad$ CCA intraductal staging & $2(4.7)$ \\
SpyGlass generation, $n(\%)$ & \\
$\quad$ SpyGlass ${ }^{\text {TM }}$ first generation & $7(16.3)$ \\
$\quad$ SpyGlass \\
PM DS & $36(83.7)$ \\
Previous ERCP, $n$ (\%) & $17(39.5)$ \\
Postprocedural complications, $n(\%)$ & $8(18.6)$ \\
$\quad$ Pancreatitis & $5(11.6)$ \\
$\quad$ Cholangitis & $3(7.0)$ \\
Follow-up after SpyGlass, days, & \\
$\quad$ mean \pm SD (minimum-maximum) & $265 \pm 311(1-1,329)$ \\
\hline
\end{tabular}

CCA, cholangiocarcinoma; ERCP, endoscopic retrograde cholangiopancreatography.

Table 2. Patients' final diagnosis

\begin{tabular}{lr}
\hline Total & $n=43, n(\%)$ \\
\hline Final diagnosis & \\
Malignant disease & \\
CCA & $24(55.8)$ \\
$\quad$ Distal CCA & $5(20.8)$ \\
$\quad$ Perihilar CCA & $19(79.2)$ \\
$\quad$ Both bile duct extension & $11(57.9)$ \\
$\quad$ Left hepatic bile duct extension & $4(21.1)$ \\
$\quad$ Right hepatic bile duct extension & $2(10.5)$ \\
$\quad$ Without intrahepatic extension & $2(10.5)$ \\
Benign disease & $19(44.2)$ \\
Extrahepatic stricture & $11(25.6)$ \\
Intrahepatic stricture & $2(4.7)$ \\
Primary sclerosing cholangitis & $1(2.3)$ \\
Caroli disease & $1(2.3)$ \\
Hypertensive biliopathy & $2(4.7)$ \\
Normal & $2(4.7)$ \\
\hline
\end{tabular}

CCA, cholangiocarcinoma.

Cholangioscopy findings revealed 10 distal lesions (23.3\%) and 28 perihilar lesions (65.1\%). In 5 cases (11.6\%), SpyGlass cholangioscopy revealed no stenosis. In these cases, the final diagnosis was primary sclerosing cholangitis $(n=1)$ and hypertensive biliopathy $(n=2)$, while the remaining 2 lesions had no significant pathological findings.

Excluding the 2 patients with a previous diagnosis of CCA, findings classified as malignant based on visual impression were observed in $22(53.7 \%)$, while the remaining $19(46.3 \%)$ were classified as benign.

\section{SpyBite Biopsies and Final Diagnosis}

Biopsy samples were obtained using SpyBite forceps under direct visualization in all patients even if no suspicious lesions were identified on cholangioscopy. A total of 16 biopsy (37.2\%) samples revealed malignant lesions, $14(32.6 \%)$ revealed benign lesions, while $13(30.2 \%)$ were inconclusive for malignancy.

Final diagnosis was made on the basis of cholangioscopy findings in 36 patients (83.7\%), surgical findings in 1 patient $(2.3 \%)$, diagnostic laparoscopy with biopsy samples in 2 patients (4.7\%), or clinical follow-up of $>6$ months in 4 patients (9.3\%). Final diagnosis was benign in 19 patients (44.2\%) and malignant in 24 patients (55.8\%). In the latter, 5 lesions were distal CCA and 19 were perihilar CCA. Of the perihilar lesions, 2 lesions did not have intrahepatic extension, 2 had extension to the right hepatic bile duct, 4 had extension to left hepatic bile duct, and 11 extended to both hepatic bile ducts (Table 2).

\section{Visual Accuracy of SpyGlass}

The diagnostic accuracy of the POC visual impression as a predictor of a malignancy was assessed in 41 patients and is described in Table 3 . Of the 22 patients with a final diagnosis of malignancy, POC impression was malignant in all of the 22 patients ( $100 \%$ sensitivity). Of 19 patients with a final diagnosis of benignity, cholangioscopic impression was malignant in 2 patients and benign in 17 patients (89.5\% specificity).

The overall accuracy of SpyGlass visual impression in differentiating malignant from benign ductal lesions was 95.1\%.

\section{SpyBite Biopsies Accuracy}

The diagnostic accuracy of the SpyBite biopsies to determine the malignant or benign nature of intraductal lesions was assessed in 41 patients and is described in Table 4. Of 22 patients with a final diagnosis of malignancy, SpyBite specimens yielded malignant histology in 14 patients (63.6\% sensitivity). Of 19 patients with a final diagnosis of benignity, SpyBite biopsies yielded benign histology in 19 patients (100\% specificity).

The overall accuracy of SpyBite biopsies in differentiating malignant from benign ductal lesions was $80.5 \%$. 
Table 3. Diagnostic accuracy of visual impression

\begin{tabular}{|c|c|c|c|c|}
\hline & \multicolumn{2}{|c|}{ Diagnosis of malignancy } & \multirow[t]{2}{*}{ Total } & \multirow[t]{2}{*}{ Predictive value } \\
\hline & yes & no & & \\
\hline \multicolumn{5}{|c|}{ Visual impression, $n$} \\
\hline Malignant & 22 & 2 & 24 & PPV 91.7\% \\
\hline Benign & 0 & 17 & 17 & NPV 100\% \\
\hline Total, $n$ & 22 & 19 & 41 & \\
\hline Diagnostic yield & Sensitivity $100 \%$ & Specificity $89.5 \%$ & Accuracy $95.1 \%$ & \\
\hline
\end{tabular}

Table 4. Diagnostic accuracy of SpyBite biopsies

\begin{tabular}{lllll}
\hline & \multicolumn{2}{l}{ Diagnosis of malignancy } & Total & Predictive value \\
\cline { 2 - 4 } & yes & no & & \\
\hline Biopsies, $n$ & & & 14 & PPV $100 \%$ \\
$\quad$ Positive & 14 & 0 & 27 & NPV $70.4 \%$ \\
\hline Negative & 8 & 19 & 41 & \\
\hline Total, $n$ & 22 & 19 & Accuracy $80.5 \%$ & \\
\hline Diagnostic yield & Sensitivity $63.6 \%$ & Specificity $100 \%$ & & \\
\hline
\end{tabular}

PPV, positive predictive value; NPV, negative predictive value.

\section{SpyGlass Preoperative Mucosal Cancerous Extension}

Of the 24 patients with a final diagnosis of CCA, 5 (20.8\%) were distal CCA and 19 (79.2\%) were perihilar.

Location of distal CCA visualized by POC procedure was in concordance to location seen in previous imaging studies. For preoperative evaluation of mucosal cancerous extension with BCC, we excluded these 5 patients with distal CCA. BCC was established in 19 lesions based on previous imagiologic intraductal extension of the tumor and on cholangioscopy findings.

In 11 patients (57.8\%), BCC with imagiologic findings was identical to cholangioscopy classification.

In the other 8 lesions, SpyGlass visual findings revealed a more extensive lesion than previously suspected. In 2 cases, a resectable BCC type 2 was a type 3 a based on SpyGlass examination, which modified the surgical approach to these patients from a bile duct resection (BDR) to a BDR with right hepatic lobectomy (Fig. 2). In 3 cases, a previous BCC type 2 lesion was reclassified as BCC type 4 on SpyGlass approach. In one of these cases, although TNM classification was Ib, the lesion was considered irresectable after POC examination. The other 2 were con- sidered irresectable due to vascular invasion. The remaining 3 patients were previously classified as a BCC type 3a based on imagiologic findings and were actually BCC type 4 on SpyGlass examination. One patient previously considered candidate for surgical resection was deemed irresectable after POC examination due to extensive biliary involvement. The other 2 were considered irresectable and were referred for palliative biliary stent. Besides biliary stent, 1 patient had additional treatment with intraductal radiofrequency ablation.

The 5 patients submitted to surgical treatment had an R0 resection with no need for adjuvant chemotherapy. Table 5 provides detailed information of specific BBC and therapeutic approach in these cases.

\section{Discussion}

In the present study, we assessed the diagnostic accuracy of the SpyGlass system as well as its capability in detecting mucosal CCA extension preoperatively. An initial prospective observational feasibility study demonstrated 

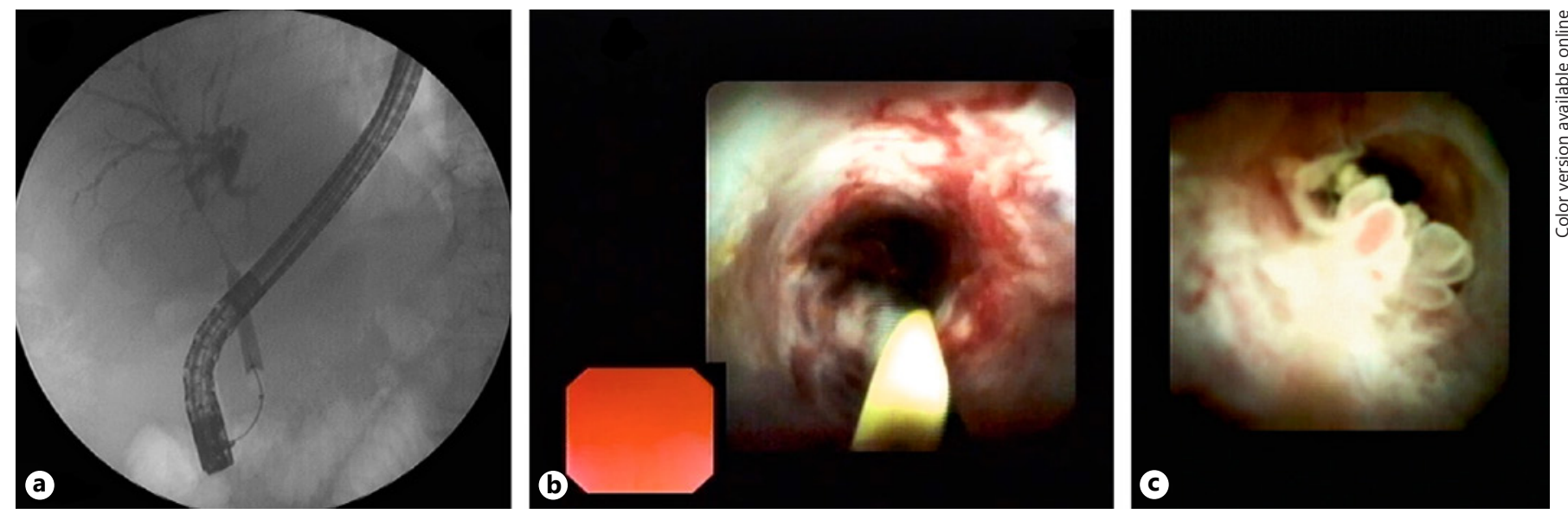

Fig. 2. a Cholangiographic view of a subhilar stenosis in a patient with presumed resectable BBC type 2. b SpyGlass showing an infiltrative stenosis with irregular and dilated vessels suggestive of malignancy. c SpyGlass showing mucosal cancerous extension into the right duct.

Table 5. Preoperative BCC and therapeutic approach

\begin{tabular}{|c|c|c|c|c|}
\hline $\begin{array}{l}\text { Patients with perihilar } \\
\text { CCA }(n=19)\end{array}$ & $\begin{array}{l}\text { Previous } \\
\text { imagiologic BCC }\end{array}$ & $\begin{array}{l}\text { SpyGlass } \\
\text { BCC }\end{array}$ & TNM & Therapeutic approach \\
\hline \multicolumn{5}{|c|}{ Lesions with same BCC $(\mathrm{n}=11 ; 57.9 \%)$} \\
\hline$n=1$ & 1 & 1 & 1. Ib & 1. NACT + surgery \\
\hline$n=1$ & 2 & 2 & 2. IV & 2. PBS \\
\hline \multirow[t]{4}{*}{$n=4$} & $3 b$ & $3 b$ & 3. IIa & 3. Surgery \\
\hline & & & 4. III & 4. $\mathrm{PBS}+\mathrm{PCT}$ \\
\hline & & & 5. IV & 5. PBS \\
\hline & & & 6. IV & 6. PBS \\
\hline \multirow[t]{5}{*}{$n=5$} & 4 & 4 & 6. Ia & 6. Surgery \\
\hline & & & 7. III & 7. PBS \\
\hline & & & 8. IV & 8. PBS + PQT \\
\hline & & & 9. IV & 9. PBS + PQT \\
\hline & & & 10. IV & 10. $\mathrm{PBS}+\mathrm{PQT}$ \\
\hline \multicolumn{5}{|c|}{ Lesions with different BCC after cholangioscopy $(\mathrm{n}=8 ; 42.1 \%)$} \\
\hline \multirow[t]{2}{*}{$n=2$} & 2 & $3 \mathrm{a}$ & 11. Ia & 11. Surgery $(\mathrm{BDR}+\mathrm{RHL})$ \\
\hline & & & 12. Ia & 12. Surgery $(\mathrm{BDR}+\mathrm{RHL})$ \\
\hline \multirow[t]{3}{*}{$n=3$} & 2 & 4 & 13. Ib & 13. PBS \\
\hline & & & 14. III & 14. $\mathrm{PBS}+\mathrm{PCT}$ \\
\hline & & & 15. III & 15. PBS + PCT \\
\hline \multirow[t]{3}{*}{$n=3$} & $3 a$ & 4 & 16. Ib & 16. $\mathrm{PBS}+\mathrm{RFA}$ \\
\hline & & & 17. III & 17. PBS \\
\hline & & & 18. IV & 18. $\mathrm{PBS}+\mathrm{PCT}$ \\
\hline
\end{tabular}

BCC, Bismuth-Corlette classification; NACT, neoadjuvant chemotherapy; PBS, palliative biliary stent; PCT, palliative chemotherapy; RFA, radiofrequency ablation; BDR, bile duct resection; RHL, right hepatic lobectomy.

that the SGDVS system can provide adequate samples for histologic diagnosis in indeterminate stenosis and to successfully guide stone therapy [6]. Subsequently, Chen et al. [7] conducted a larger scale multicenter prospective observational study in 297 patients with biliary strictures and/or stones; SGDVS visual impression had a sensitivity, specificity, positive predictive value, and negative predictive value for diagnosing malignancy of $78,82,80$, and $80 \%$, respectively. For SGDVS-directed biopsy, the respective results were $49,98,100$, and $72 \%$. 
Using the Xie et al. [11] classification, the visual impression of malignancy was in concordance with final diagnosis in all cases, with a $100 \%$ sensitivity and an $89.5 \%$ specificity in differentiating malignant from benign lesions. Furthermore, targeted biopsies with SpyBite forceps revealed, in our study, $63.6 \%$ sensitivity and $100 \%$ specificity in differentiating malignant from benign lesions. According to a systematic review published in 2015, pooled sensitivity for detecting malignant bile duct lesion by cholangioscopy with targeted biopsies was $66.1 \%$ and specificity was $98.0 \%$ [13], which was similar to our results. Using the new digital system (SpyGlass DS) Navaneethan et al. [14] reported sensitivity of $90.0 \%$ and specificity of $95.8 \%$ for diagnosis of malignancy based on visual impression. The sensitivity and specificity of cholangioscopy-targeted biopsies was 85 and 100\%, respectively. The reduced sensitivity of cholangioscopic targeted biopsies may be explained by the difficulty of obtaining representative material with the miniature forceps used, although the observed lesion is highly suspected of malignancy. A significant number of our SpyBite biopsies remained inconclusive (30.2\%). Since the specimens collected are small, the histopathological examination may be difficult to interpret even for experienced anatomopathologists. With acquired experience, it is expected that these results will improve even in difficult cases such as strictures in the context of primary sclerosing cholangitis. Added to the fact that a review of 16 studies evaluating sensitivity of ERCP brush cytology [15] found an overall sensitivity of $41.6 \pm 3.2 \%(99 \% \mathrm{CI})$ with a negative predictive value of $58.0 \pm 3.2 \%(99 \% \mathrm{CI})$, our results corroborate cholangioscopy as a promising procedure in improving the sensitivity for the diagnosis of malignant biliary strictures.

Our findings show that visual impression has a higher diagnostic yield than targeted biopsy, which is in concordance with published data [16]. In clinical practice, these data construe the fact that despite cholangioscopy favors visual identification of a malignant lesion, it still cannot demonstrate with a high diagnostic accuracy that this lesion is malignant histologically. Further improvements in biopsy approach, namely on SpyBite forceps, are necessary.

A recent report compared first-generation Spy Glass ${ }^{\mathrm{TM}}$ with SpyGlass ${ }^{\mathrm{TM}}$ DS in a retrospective database involving 8 years [17]. Introduction of the digital version increased the utilization of single operator cholangioscopy compared to the fiberoptic $(p=0.0001)$. Moreover, the overall success of diagnostic cholangioscopy was marginally higher for the SpyGlass ${ }^{\mathrm{TM}}$ DS than for first-generation
SpyGlass ${ }^{\mathrm{TM}}(p=0.07)$. Essential difference of the latest version is its improved ease of setup, ease of use, and quality imaging, with a higher angle yield. In our experience, the major advantages of the SpyGlass ${ }^{\mathrm{TM}}$ DS are the image quality, which facilitates morphological identification of the lesions, namely, the identification of tumor vessels, and the shorter duration of the procedure.

Curative operative procedure in perihilar CCA aims to achieve an R0 tumor resection [18]. Tumor staging, not only of the vascular extension but also of the intraductal extension evaluated in BCC, is essential to achieve this purpose. SGDVS allowed evaluation and classification of the 19 perihilar CCA. The feasibility of SpyGlass in this context has been reported, providing evidence that this technique can accurately evaluate longitudinal extension of the tumor. In a pilot study, Ogawa et al. [19] evaluated the usefulness of SGDVS-guided mapping biopsy for preoperative evaluation of extrahepatic CCA. The overall success rate of SGDVS-guided biopsy and diagnostic accuracy was $88 \%$. Although the number of patients evaluated was small $(n=13)$, the authors concluded that SGDVS was feasible for preoperative evaluation of extrahepatic CCA. Another recent small report [20] evaluated the feasibility and the safety of intraoperative ERCP and SGDVS to assess the intraductal border of CCA proliferation. Based on intraoperative SGDVS, only 2 of the 14 patients included were indicated for resection, and in both cases, R0 resection was achieved. A multicenter study [21], including 118 patients with pancreatobiliary lesions, SGDVS changed the surgical plan in 39 (34\%) patients: 8 of 13 in the pancreatic duct and 32 of 105 in the bile duct. In the bile duct, 6 patients (5\%) had less extensive surgery and 26 patients (25\%) avoided surgery.

Despite our small sample size, cholangioscopy approach altered previous imagiologic BCC in $42.1 \%(n=$ 8 ) and changed surgical management in $21 \%(n=4)$ of the patients. Surgery was avoided in 2 previously considered resectable lesions due to extensive intraductal involvement and detection of more extensive biliary involvement than previously suspected modified 2 surgical approaches (from a simple BDR to a BDR with right hepatic lobectomy). All patients deemed candidate for surgery after cholangioscopic evaluation achieved and R0 resection. Similar results were recently reported evaluating the role of SGDVS in the presurgical assessment of CCA resectability [20]. Our results suggest that SGDVS can detect intraductal mucosal cancer extension not evident in imagiologic studies. This information is important for delineating surgical treatment and avoiding surgery in patients with extensive biliary involvement. 
Cost-effectiveness of SpyGlass procedure needs to be in count, even though few studies approach this subject. According to Deprez et al. [22], the use of cholangioscopy in biliary stricture diagnosis determined a decrease in the number of procedures $(-31 \%)$ and costs (EUR -13,000) when compared to ERCP. Although lack of clinical trials limits conclusions on this topic, our preliminary findings suggest that cholangioscopy could have an important role as a first-line procedure for biliary diagnosis and staging of potentially resectable CCA. Combining imagiologic vascular evaluation with cholangioscopy can optimize surgery results and reduced associated costs.

Our study has some limitations, namely, its retrospective nature with evaluation of only 43 patients. In addition, this study was not randomized, and most of the patients had already received imaging modalities and ERCP prior to the cholangioscopy procedure, which raises concerns about patient selection bias. Besides, we based SpyGlass visual evaluations on the assessment done by 2 experienced endoscopists according to previously published criteria [11] and found a good agreement with SpyBite-targeted biopsy results, but validation of classification criteria needs further studies.

Registered AEs were observed in $18.6 \%$ of the procedures. Although this number may be slightly superior to traditional ERCP, we did not observed any serious AEs associated with POC performance. A possible explanation for an increased number of AEs in this patient group may be that these procedures tend to be longer, resulting in more intraluminal air insufflation and distension of the bile duct during irrigation [23]. With accumulating experience and improved image quality of Spy Glass ${ }^{\mathrm{TM}} \mathrm{DS}$, the need for irrigation diminished and this may decrease the rate of AEs.
In conclusion, our results support latest findings on the utility of SpyGlass ${ }^{\mathrm{TM}}$ system in the diagnosis of biliary stenosis. Its use in potentially CCA resectable cases was important to establish the best therapeutic approach. In the future, preoperative staging of perihilar CCA with POC combined with imagiologic evaluation of lesions vascular extension may optimize surgical results.

\section{Acknowledgment}

None to declare.

\section{Statement of Ethics}

The research was conducted ethically in accordance with the World Medical Association Declaration of Helsinki.

\section{Disclosure Statement}

The authors have no conflicts of interest to declare.

\section{Funding Sources}

There is no funding sources to declare.

\section{Author Contributions}

P.P.: performed the majority of endoscopic procedures, writing of the manuscript. S.S.: data collection and writing of the manuscript. R.M.: data collection and statistical analysis. R.G.: data collection. E.R.-P. and F.V.-B.: performed the procedures, critical revision of the manuscript. G.M.: critical revision and final approval of the manuscript.

\section{References}

1 Parsi MA. Common controversies in management of biliary strictures. World J Gastroenterol. 2017 Feb;23(7):1119-24.

2 Kapoor BS, Mauri G, Lorenz JM. Management of Biliary Strictures: State-of-the-Art Review. Radiology. 2018 Dec;289(3):590603.

3 Blechacz B. Cholangiocarcinoma: Current Knowledge and New Developments. Gut Liver. 2017 Jan;11(1):13-26.

4 Navaneethan U, Njei B, Lourdusamy V, Konjeti R, Vargo JJ, Parsi MA. Comparative effectiveness of biliary brush cytology and intraductal biopsy for detection of malignant biliary strictures: a systematic review and meta-analysis. Gastrointest Endosc. 2015 Jan;81(1):16876.

5 Bowlus CL, Olson KA, Gershwin ME. Evaluation of indeterminate biliary strictures. Nat Rev Gastroenterol Hepatol. 2017 Dec; 14(12): 749.

6 Chen YK, Pleskow DK. SpyGlass single-operator peroral cholangiopancreatoscopy system for the diagnosis and therapy of bile-duct disorders: a clinical feasibility study (with video). Gastrointest Endosc. 2007 May;65(6):832-41.

7 Chen YK, Parsi MA, Binmoeller KF, Hawes RH, Pleskow DK, Slivka A, et al. Single-oper- ator cholangioscopy in patients requiring evaluation of bile duct disease or therapy of biliary stones (with videos). Gastrointest Endosc. 2011 Oct;74(4):805-14.

8 Pereira P, Vilas-Boas F, Peixoto A, Andrade P, Lopes J, Macedo G. How SpyGlass ${ }^{\mathrm{TM}}$ May Impact Endoscopic Retrograde Cholangiopancreatography Practice and Patient Management. GE Port J Gastroenterol. 2018 Apr; 25(3):132-7.

9 Bismuth H, Corlette MB. Intrahepatic cholangioenteric anastomosis in carcinoma of the hilus of the liver. Surg Gynecol Obstet. 1975 Feb;140(2):170-8. 
10 Cotton PB, Eisen GM, Aabakken L, Baron $\mathrm{TH}$, Hutter MM, Jacobson BC, et al. A lexicon for endoscopic adverse events: report of an ASGE workshop. Gastrointest Endosc. 2010 Mar;71(3):446-54.

11 Xie C, Aloreidi K, Patel B, Ridgway T, Thambi-Pillai $T$, Timmerman $G$, et al. Indeterminate biliary strictures: a simplified approach. Expert Rev Gastroenterol Hepatol. 2018 Feb; 12(2):189-99.

12 Chun YS, Pawlik TM, Vauthey JN. 8th Edition of the AJCC Cancer Staging Manual: Pancreas and Hepatobiliary Cancers. Ann Surg Oncol. 2018 Apr;25(4):845-7.

13 Navaneethan U, Hasan MK, Lourdusamy V, Njei B, Varadarajulu S, Hawes RH. Single-operator cholangioscopy and targeted biopsies in the diagnosis of indeterminate biliary strictures: a systematic review. Gastrointest Endosc. 2015 Oct;82(4):608-14.e2.

14 Navaneethan U, Hasan MK, Kommaraju K, Zhu X, Hebert-Magee S, Hawes RH, et al. Digital, single-operator cholangiopancreatoscopy in the diagnosis and management of pancreatobiliary disorders: a multicenter clinical experience (with video). Gastrointest Endosc. 2016 Oct;84(4):649-55.
15 Burnett AS, Calvert TJ, Chokshi RJ. Sensitivity of endoscopic retrograde cholangiopancreatography standard cytology: 10-y review of the literature. J Surg Res. 2013 Sep;184(1): 304-11.

16 Korrapati P, Ciolino J, Wani S, Shah J, Watson R, Muthusamy VR, et al. The efficacy of peroral cholangioscopy for difficult bile duct stones and indeterminate strictures: a systematic review and meta-analysis. Endosc Int Open. 2016 Mar;4(3):E263-75.

17 Dimas ID, Vardas E, Papastergiou V, Fragaki M, Velegraki M, Mpitouli A, et al. Comparison of digital versus fiberoptic cholangioscopy in patients requiring evaluation of bile duct disease or treatment of biliary stones. Ann Gastroenterol. 2019 Mar-Apr;32(2): 199-204.

18 Lewis HL, Rahnemai-Azar AA, Dillhoff M, Schmidt CR, Pawlik TM. Current Management of Perihilar Cholangiocarcinoma and Future Perspectives. Chirurgia (Bucur). 2017 May-Jun;112(3):193-207.

19 Ogawa T, Ito K, Koshita S, Kanno Y, Masu K, Kusunose $\mathrm{H}$, et al. Usefulness of cholangioscopic-guided mapping biopsy using SpyGlass DS for preoperative evaluation of extra- hepatic cholangiocarcinoma: a pilot study. Endosc Int Open. 2018 Feb;6(2):E199-204.

20 Hajer J, Havlůj L, Whitley A, Oliverius M, Gürlich R. The role of single-operator cholangioscopy (SpyGlass) in the intraoperative diagnosis of intraductal borders of cholangiocarcinoma proliferation - pilot study. Cas Lek Cesk. 2019;158(2):68-72.

21 Tyberg A, Raijman I, Siddiqui A, Arnelo U, Adler DG, Xu MM, et al. Digital Pancreaticocholangioscopy for Mapping of Pancreaticobiliary Neoplasia: Can We Alter the Surgical Resection Margin? J Clin Gastroenterol. 2019 Jan;53(1):71-5.

22 Deprez PH, Garces Duran R, Moreels T, Furneri G, Demma F, Verbeke L, et al. The economic impact of using single-operator cholangioscopy for the treatment of difficult bile duct stones and diagnosis of indeterminate bile duct strictures. Endoscopy. 2018 Feb; 50(2):109-18.

23 Maple JT, Keswani RN, Hovis RM, Saddedin EZ, Jonnalagadda S, Azar RR, et al. Carbon dioxide insufflation during ERCP for reduction of postprocedure pain: a randomized, double-blind, controlled trial. Gastrointest Endosc. 2009 Aug;70(2):278-83. 